- 研究报告・

\title{
舟山群岛蝶类群落嵌套分布格局及其影响因素
}

\author{
张雪梅 ${ }^{1} \quad$ 韩徐芳 $^{1} \quad$ 刘立伟 $^{2} \quad$ 徐爱春 $^{1 *}$ \\ 1 (中国计量大学生命科学学院, 杭州 310018) \\ 2 (浙江自然博物馆, 杭州 310012)
}

\begin{abstract}
摘要: 片断化生境中群落的物种组成常呈现嵌套分布格局。2013年7-8月, 我们在浙江舟山群岛采用截线法对 28 个岛屿上的蝴蝶群落进行了野外调查, 探讨了岛屿物种嵌套分布格局及其影响因素。通过测量采集标本获得蝶类 的生活史特征(最小需求面积、翅展和体重), 查阅文献资料获得蝶类的栖息地特征(岛屿面积、距最近大陆距离和 距最近大岛距离), 分析了影响蝶类群落嵌套结构的因素。研究结果显示: (1)舟山群岛蝶类群落符合嵌套分布格局; (2)岛屿面积和物种最小需求面积对嵌套格局的形成有显著影响; (3)舟山群岛蝶类群落嵌套格局的形成支持选择 性灭绝假说; (4)随机检验零模型结果显示该嵌套分布格局并非采样偏差造成的。因此，在制定舟山群岛区域蝶类 保护措施时，应优先考虑那些分布在面积较大岛屿的和最小需求面积较大的物种。
\end{abstract}

关键词: 栖息地片断化; 嵌套结构; 选择性灭绝; 蝶类; 舟山群岛

\section{Influencing factors of the nested distribution of butterfly assemblages in the Zhoushan Archipelago, China}

\author{
Xuemei Zhang ${ }^{1}$, Xufang $\operatorname{Han}^{1}$, Liwei Liu ${ }^{2}$, Aichun $\mathrm{Xu}^{1 *}$ \\ 1 College of Life Sciences, China Jiliang University, Hangzhou 310018 \\ 2 Zhejiang Museum of Natural History, Hangzhou 310012
}

\begin{abstract}
In natural ecosystem, species assemblages in fragmented habitats frequently exhibit a nested subset pattern. Although nestedness has been documented for a wide range of taxa, it is rarely tested in butterfly assemblages in oceanic archipelago ecosystems. We surveyed the butterfly occupancy and abundance on 28 islands using a line-transect method in the Zhoushan Archipelago, China from July to August 2013. We determined island geographical factors (area, isolation) based on the literature and obtained species life-history traits (area requirement, wingspan and body weight) in the laboratory. The recently developed metric WNODF was used to examine the nestedness. Partial Spearman rank correlation was used to evaluate the associations of nestedness and island geographical factors as well as life-history traits related to species extinction and immigration tendencies. We found that the community composition of butterflies in the Zhoushan Archipelago was significantly nested. Island area and species' area requirements were significantly correlated with nestedness after synthesis for all independent variables. In contrast, according to the null-model random test, nestedness of butterflies might not result from passive sampling or selective colonization. Our results indicate that selective extinction is the main driver of nestedness of butterflies in our system. From a conservation perspective, we suggest that large islands and species with large area requirements should be paid more attention in order to maximize the total number of species preserved.
\end{abstract}

Key words: habitat fragmentation; nestedness; selective extinction; butterfly; Zhoushan Archipelago

栖息地片断化(habitat fragmentation)是导致生 物多样性丧失的主要原因之一(McGarigal \&
Cushman, 2002; Gibson et al, 2013)。研究表明，在片 断化栖息地系统中群落物种组成常呈现嵌套分布

收稿日期: 2015-11-13; 接受日期: 2016-01-10

基金项目: 国家自然科学基金(31272325)和浙江省自然科学基金(Y5090080)

" 通讯作者 Author for correspondence. E-mail: springlover@cjlu.edu.cn 
格局(nestedness) (Soga \& Koike, 2012)。Darlington (1957)最早提出嵌套格局的概念, 即描述一种在低 丰富度斑块中出现的物种大多数也在丰富度相对 较高的斑块中出现的非随机分布模式。Patterson和 Atmar (1986)首次系统地提出了在群落水平构建矩 阵进行嵌套分析的方法, 使嵌套格局理论在群落生 态学领域被广泛应用(Ulrich et al, 2009)。随着研究 方法的不断发展(Rodríguez-Gironés \& Santamaría, 2006; Almeida-Neto \& Ulrich, 2011), 嵌套理论在研 究片断化栖息地中物种组成和分布模式中发挥着 越来越重要的作用(Simaiakis \& Martínez-Morales, 2010), 并已逐渐成为群落形成机制研究的重要工 具(Lindo et al, 2008; Florencio et al, 2011)。

关于嵌套格局的形成原因主要有 4 种假说(陈水 华和王玉军, 2004; Wang et al, 2010), 即选择性灭绝 假说(the selective extinction hypothesis)、选择性迁入 假说(the selective colonization hypothesis)、生境嵌套 假说(the habitat nestedness hypothesis)和被动抽样假 说(the passive sampling hypothesis)。选择性灭绝假 说认为斑块面积制约物种分布, 最小需求面积较大 的物种其灭绝风险也较高, 将率先从较小面积的斑 块化栖息地中灭绝，因此形成嵌套格局(Menezes \& Fernandez, 2013; Matthews et al, 2015)。选择性迁入 假说认为由于物种扩散能力的差异, 迁移能力或强 或弱的物种都可占据隔离度较小的斑块, 而扩散能 力强的少数物种则可占据隔离度较大的斑块, 进而 形成嵌套格局(Hill et al, 2011; Habel et al, 2013)。 生境嵌套假说认为物种分布与生境密切相关, 物种 分布的嵌套格局是斑块间生境结构呈嵌套分布的 结果(Li et al, 2013; Wang et al, 2013)。被动抽样假说 基于物种间多度差异, 在抽取样本时, 多度高的物 种被抽中的概率大; 在不同取样面积下，多度高的 物种不论是在小面积还是在大面积中抽样出现的 概率都大, 从而形成嵌套格局(McQuaid \& Britton, 2013)。探讨物种嵌套格局及其形成机制，对嵌套结 构及其理论的发展, 以及将其应用于野生动物的保 护和管理具有重要的指导意义(Matthews et al, 2015)。

目前，嵌套分析被广泛应用于各种斑块化栖息 地中群落组成和分布格局的研究, 如陆桥岛屿 (land-bridge islands) (Wang et al, 2010, 2012; Hu et al, 2011)、片断化森林斑块 (fragmented forest patches) (Hill et al, 2011)和海洋性群岛 (oceanic archipelagos) (Dennis et al, 2012)等。涉及的动物类 群也十分丰富, 包括哺乳类(Rodríguez \& Ojeda, 2013)、鸟类(Wang et al, 2013)和两栖爬行类( Li et al, 1998; Wang et al, 2012)等。蝶类作为重要的传粉昆 虫和环境指示昆虫, 具有观赏、科研等多方面价值, 在生态系统中占据不可替代的地位(周尧, 2000), 探 讨其群落组成和分布结构的特征对蝶类的保护非 常重要。已有研究表明, 在多数情况下面积和生境 类型等栖息地特征参数是影响蝶类嵌套结构的主 要因素(Öckinger \& Smith, 2006; Soga \& Koike, 2012); 同时, Dennis等(2012)的研究结果指出, 与蝶 类扩散能力和灭绝倾向相关的生活史特征参数也 可影响嵌套结构的形成。然而, 综合栖息地特征和 物种生活史特征两方面因素，在特定栖息地类型 (如海洋性陆桥岛)中对物种嵌套分布结构的研究还 很少。

舟山群岛是我国最大的群岛, 形成于晚更新世 时期(Wang et al, 2014), 以海水为单一隔离介质, 片 断化时间长 (至今约 7,000-9,000年), 各岛屿物种分 布已达稳定, 是研究栖息地片断化理论及物种嵌套 分布格局的自然实验系统。2013年7-8月, 我们在舟 山群岛以蝶类为研究对象, 对以下 3 个科学问题进 行了探讨: (1)检验舟山群岛蝶类群落分布是否符合 嵌套分布格局; (2)分析岛屿面积、隔离度等栖息地 特征以及蝶类最小需求面积、翅展和体重等物种生 活史特征对嵌套结构的影响; (3)探讨舟山群岛蝶类 群落嵌套分布格局的形成机制。通过以上研究，揭 示栖息地片断化对蝶类物种多样性和群落结构的 影响机制, 并为片断化栖息地中蝶类物种保护策略 的制定提供理论依据。

\section{1 研究地点}

舟山群岛 $\left(29^{\circ} 32^{\prime}-31^{\circ} 04^{\prime} \mathrm{N}, 121^{\circ} 30^{\prime}-123^{\circ} 25^{\prime} \mathrm{E}\right)$ 位于长江口以南、杭州湾以东的浙江省东北部海域, 属典型的陆桥岛屿, 其陆域系浙东天台山山脉向东 海逶迤延伸而成，境内岛屿呈东北-西南向排列。本 区属北亚热带南缘季风海洋性气候, 温暖湿润, 年 均气温 $15.4-16.7^{\circ} \mathrm{C}$, 年均降雨量 $850.6-1,367.1 \mathrm{~mm}$, 雨量充沛, 四季分明。植被主要为亚热带常绿榈叶 林。地形与邻近大陆相似, 其中丘陵约占 $70 \%$, 平原 约占30\% (周航等, 1998; Wang et al, 2014)。 


\section{2 方法}

\section{1 样地设置}

根据岛屿面积(不含潮间带)大小及其与大陆间 的隔离度梯度, 在舟山群岛选取 28 个岛屿作为研究 样地。将岛屿按面积从大到小的顺序依次标记为 1-28号, 其中 25-28号岛屿无人居住, 其他岛屿有 居民长期居住。岛屿景观参数及方位分别见表 1 、 图1。

\section{2 蝶类种类调查}

选择无强风(3级以下, 风力等级标准参考 $\mathrm{GB} / \mathrm{T}$ 28591-2012风力等级)、无雾和无雨天气, 采用截线 法(line-transect method) 对蝶类进行野外调查和样本 采集(Benedick et al, 2006; Pe'er et al, 2011)。调查前 从“舟山天地图”(http://www.zsch.gov.cn/main.aspx) 下载各岛屿卫星地图以获取岛上生境分布的基本 信息，排除调查者无法抵达的地点后设置样线以覆 盖所有生境类型。样线采用分层设置: 岛屿面积大 于 2,000 ha (1-10号岛)的设置 8 条样线, 每条样线长 $2 \mathrm{~km}$; 面积在100-2,000 ha之间(11-23号岛)的设置4 条样线, 每条样线长 $1.5 \mathrm{~km}$; 面积小于 $100 \mathrm{ha}$ (24-28号岛)的设置 2 条样线, 每条样线长 $1 \mathrm{~km}$; 每 个岛屿重复调查 2 次, 调查间隔时间为 3 天。调查选 择在7-8月间(夏季)进行，每天8:00-16:00调查者以 约 $10 \mathrm{~m} / \mathrm{min}$ 的速度沿着样线前进, 用昆虫网捕捉样 线两侧各 $5 \mathrm{~m}$ 范围内的蝶类, 处理后置入硫酸纸三 角袋中，并记录采集人、时刻和地点等。对于仅飞 过样线而未捕捉到的蝶类, 因无法准确辨认物种故

表1 舟山群岛 28 个取样岛屿的特征参数

Table 1 Characteristic parameters of the 28 sampling islands in the Zhoushan Archipelago

\begin{tabular}{|c|c|c|c|c|c|c|c|}
\hline $\begin{array}{l}\text { 岛屿编号 } \\
\text { Island code }\end{array}$ & $\begin{array}{c}\text { 岛名 } \\
\text { Island name }\end{array}$ & $\begin{array}{c}\text { 面积 } \\
\text { Area (ha) }\end{array}$ & $\begin{array}{l}\text { 距最近大陆距离 } \\
\text { Distance to the nearest } \\
\text { mainland }(\mathrm{km})\end{array}$ & $\begin{array}{l}\text { 距最近大岛距离 } \\
\text { Distance to the nearest } \\
\text { larger island }(\mathrm{km})\end{array}$ & $\begin{array}{c}\text { 物种数 } \\
\text { Number of } \\
\text { species }\end{array}$ & $\begin{array}{c}\text { 多度 } \\
\text { Abundance }\end{array}$ & $\begin{array}{l}\text { 嵌套序列 } \\
\text { Nested matrix } \\
\quad \text { rank }\end{array}$ \\
\hline 1 & 舟山 Zhoushan & $47,616.50$ & 9.10 & 9.10 & 41 & 732 & 1 \\
\hline 3 & 六横 Liuheng & $9,365.80$ & 6.97 & 6.97 & 39 & 294 & 2 \\
\hline 2 & 岱山 Daishan & $10,496.70$ & 59.42 & 11.38 & 32 & 1,102 & 3 \\
\hline 7 & 桃花 Taohua & $4,037.00$ & 9.44 & 5.98 & 32 & 432 & 4 \\
\hline 4 & 金塘 Jintang & $7,735.20$ & 3.50 & 3.50 & 29 & 410 & 5 \\
\hline 9 & 梅山 Meishan & $2,690.20$ & 0.45 & 0.45 & 29 & 146 & 6 \\
\hline 8 & 大榭 Daxie & $2,837.40$ & 0.40 & 0.40 & 23 & 163 & 7 \\
\hline 6 & 矔山 Qushan & $5,979.20$ & 55.83 & 11.38 & 22 & 555 & 8 \\
\hline 5 & 朱家尖 Zhujiajian & $6,181.50$ & 19.02 & 0.89 & 21 & 231 & 9 \\
\hline 10 & 泗礁 Sijiao & $2,135.00$ & 46.07 & 25.27 & 20 & 339 & 10 \\
\hline 12 & 长白 Changbai & $1,109.80$ & 30.38 & 1.75 & 20 & 81 & 11 \\
\hline 23 & 富翅 Fuchi & 109.10 & 19.21 & 0.61 & 19 & 124 & 12 \\
\hline 16 & 盘峙 Panzhi & 377.80 & 9.21 & 1.04 & 19 & 61 & 13 \\
\hline 15 & 嵊山 Shengshan & 422.40 & 81.26 & 25.37 & 17 & 355 & 14 \\
\hline 17 & 花鸟 Huaniao & 358.60 & 66.23 & 17.04 & 16 & 297 & 15 \\
\hline 20 & 金鸡 Jinji & 171.70 & 47.70 & 0.28 & 16 & 165 & 16 \\
\hline 19 & 蚂蚁 Mayi & 222.20 & 10.98 & 1.05 & 16 & 141 & 17 \\
\hline 24 & 西蟹峙 Xixiezhi & 82.10 & 9.92 & 0.85 & 16 & 96 & 18 \\
\hline 18 & 鲁家峙 Lujiazhi & 303.90 & 15.09 & 0.20 & 16 & 62 & 19 \\
\hline 14 & 大黄龙 Dahuanglong & 512.40 & 61.00 & 3.54 & 13 & 150 & 20 \\
\hline 11 & 虾峙 Xiazhi & $1,700.50$ & 13.89 & 2.08 & 13 & 76 & 21 \\
\hline 13 & 小长涂 Xiaochangtu & $1,092.00$ & 39.18 & 2.71 & 12 & 90 & 22 \\
\hline 21 & 白沙 Baisha & 143.90 & 30.40 & 2.45 & 11 & 37 & 23 \\
\hline 22 & 壁下 Bixia & 117.90 & 77.18 & 5.18 & 10 & 136 & 24 \\
\hline 26 & 柱住山 Zhuzhushan & 23.94 & 66.98 & 2.06 & 4 & 11 & 25 \\
\hline 25 & 东库山 Dongkushan & 24.26 & 66.64 & 3.64 & 4 & 10 & 26 \\
\hline 27 & 彩旗山 Caiqishan & 4.47 & 68.84 & 0.60 & 2 & 9 & 27 \\
\hline 28 & 台南山 Tainanshan & 2.55 & 67.30 & 3.10 & 1 & 2 & 28 \\
\hline
\end{tabular}




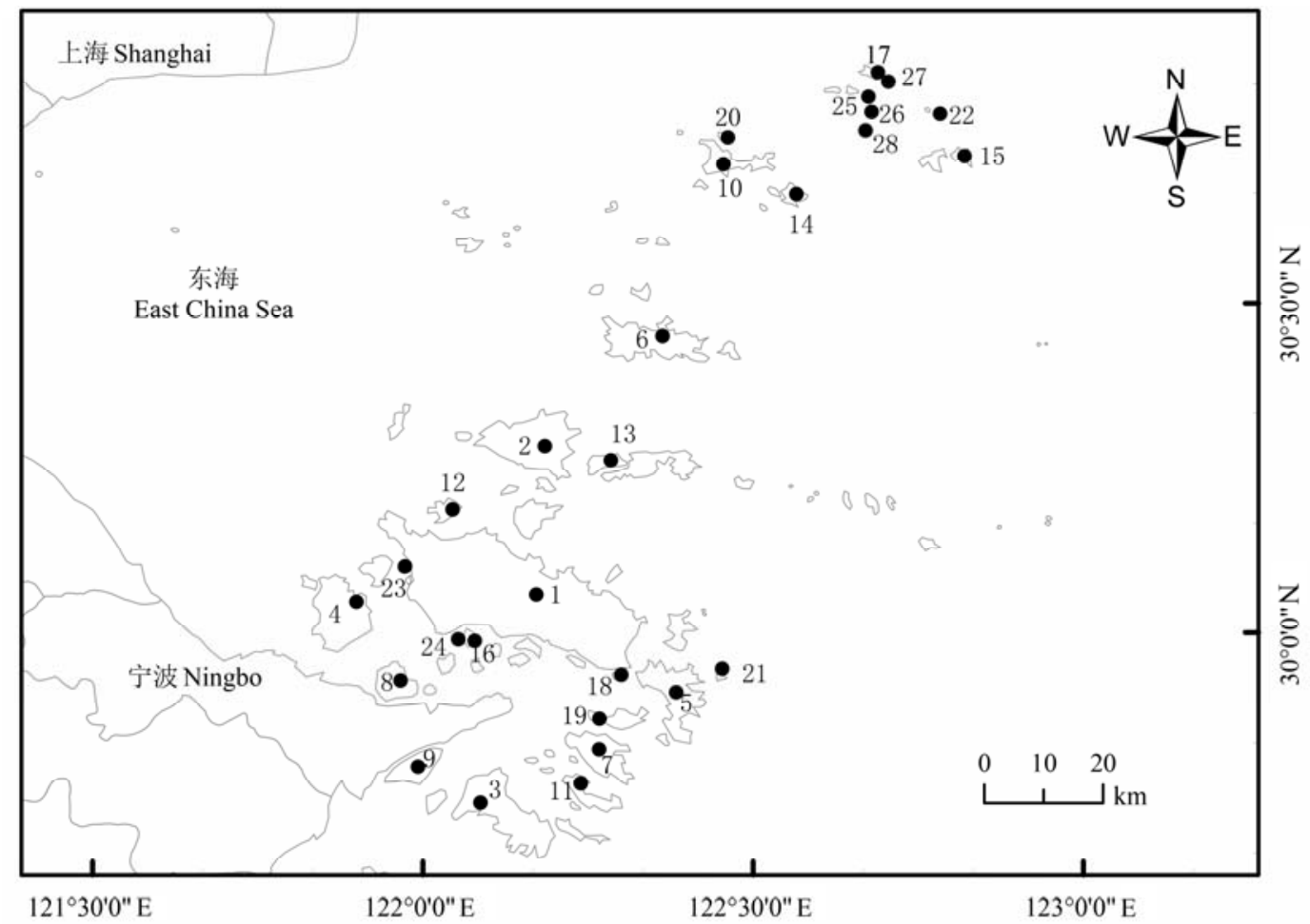

图1 舟山群岛 28 个取样岛屿方位图。岛屿编号见表1。

Fig. 1 Location of the 28 sampling islands in the Zhoushan Archipelago. The codes for all islands are shown in Table 1.

未做记录。物种命名参考《中国蝶类志》(周尧, 2000)。

\section{3 栖息地特征}

栖息地特征变量包括岛屿面积、距最近大陆距 离和距最近大岛距离。其中, “最近大岛”定义为与被 测岛屿相比面积更大且距离最近的岛(张竞成等, 2008); 距最近大陆距离和距最近大岛距离反映蝶 类从陆地或岛屿上迁入时至少需要跨越的直线距 离(Dennis et al, 2012; Wang et al, 2012)。岛屿面积 (ha)参考《浙江海岛志》(周航等, 1998), 距最近大 陆距离 $(\mathrm{km})$ 及距最近大岛距离 $(\mathrm{km})$ 参数均根据“舟 山天地图”确定(http://www.zsch.gov.cn/main.aspx)。

\section{4 生活史特征}

蝶类的生活史特征变量包括蝶类的最小需求 面积、翅展和体重。其中, 每个物种的最小需求面 积为该物种出现的最小岛屿面积(ha) (Wang et al, 2010)。使用游标卡尺 $\left[\right.$ OK-TOOLS ${ }^{\circledR}(0-15 \mathrm{~mm}$, YB5001B, 贝特, 杭州) ]测量蝶类标本翅展, 以平 均值作为翅展 $(\mathrm{mm})$ 参数。使用电子天平 $[$ 双杰 $\mathrm{G} \&$ $\mathrm{G}^{\circledR}(0-120 \mathrm{~g}, \mathrm{JJ} 124 \mathrm{BC}$, 双杰, 常熟)]称量烘干虫体,
以平均值作为体重 $(\mathrm{mg})$ 参数。

\section{5 数据处理和分析}

\subsection{1 嵌套程度量化}

构建种类-地点矩阵。矩阵中行代表物种, 列代 表研究岛屿, 以“1”表示物种出现, “ 0 ”表示物种未 出现。采用Almeida-Neto和 Ulrich (2011) 提出的 WNODF (weighted nestedness metric based on overlap and decreasing fill)嵌套分析方法来量化嵌套程 度。WNODF与其他分析方法相比具有以下优点: (1) 可利用物种多度信息进行嵌套分析; (2)对矩阵大小 和填充度不敏感; (3)不仅可以对整个矩阵进行嵌套 分析, 还可分别对物种 $\left(\mathrm{WNODF}_{\mathrm{R}}\right)$ 和研究岛屿 $\left(\mathrm{WNODF}_{\mathrm{C}}\right)$ 进行嵌套分析; (4) 能有效避免 I型错误 (Almeida-Neto \& Ulrich, 2011)。选择参数为: 行列 固定零模型(null model), 即行和列均保持原矩阵的 丰富度和多度; 输出矩阵排序原则, 即按岛屿物种 丰富度和多度对输出矩阵进行排序 (Simaiakis \& Martínez-Morales, 2010)。软件随机产生 1,000个矩 阵, 对输入矩阵进行显著性检验。使用NODF 2.0 (Almeida-Neto \& Ulrich, 2011)进行计算。 


\subsection{2 嵌套因素分析}

采用Spearman秩相关分析嵌套格局的影响因 素(Schouten et al, 2007; Wang et al, 2010)。种类-地 点矩阵经WNODF计算重排后的物种和地点排序称 嵌套序列(nested ranking)。将嵌套序列分别和各项 栖息地特征参数及物种生活史特征参数进行 Spearman秩相关分析, 检验各因素对嵌套结构的形 成是否有显著影响。由于变量间存在共线性, 采用 partial Spearman秩相关分析检验各变量对嵌套的单 独贡献(Azeria \& Kolasa, 2008; Frick et al, 2009)。使 用SAS9.2 (SAS Institute, Cary, NC, USA)进行各项 计算。

随机分布模型(random placement model)常用于 检验被动取样是否能解释嵌套格局(Andrén, 1994; Wang et al, 2012)。根据该模型计算在面积为 $a$ 的第 $k$ 个岛屿上的物种的期望值 $S_{(\alpha)}$ :

$$
\begin{aligned}
& S_{(\alpha)}=S-\sum_{i=1}^{S}(1-\alpha)^{n_{i}} \\
& \alpha=a_{k} / \sum_{k=1}^{k} a_{k} \\
& \sigma^{2}=\sum_{i=1}^{S}(1-\alpha)^{n i}-\sum_{i=1}^{S}(1-\alpha)^{2 n i}
\end{aligned}
$$

式中, $S$ 表示所有 $k$ 个研究岛屿上物种的总数, $\alpha$ 是面
积为 $a$ 的岛屿的相对面积, $n_{i}$ 表示面积为 $a$ 的第 $k$ 个岛 屿上第 $i$ 个物种的多度 $(i=1,2, \ldots, S), \sigma^{2}$ 为 $S_{(\alpha)}$ 的方 差。若有超过 $1 / 3$ 的调查点不在预测种-面积关系曲 线标准差之内, 则拒绝随机分布假说(Coleman et al, 1982; Wang et al, 2012)。

\section{3 结果}

\section{1 栖息地特征}

28 个研究岛屿面积从 2.55 到 $47,616.50 \mathrm{ha}$ 不等; 距最近大陆距离0.40-81.26 km; 距最近大岛距离 0.20-21.37 km; 各岛蝶类物种丰度从 1 到 41 种不等 (表1)。

\section{2 蝶类生活史特征}

在 28 个取样岛屿中共捕获蝶类6,307只, 隶属7 科45属64种(表2)。从获得的蝶类生活史特征参数可 知, 舟山群岛蝶类的最小需求面积从 2.55 到 47,616.50 ha不等, 翅展在11.99-106.41 mm之间, 体重在3.19-290.54 mg之间, 占据的岛屿数从 1 个至 27 个不等(表2)。

\section{3 嵌套程度量化结果}

舟山群岛蝶类群落呈现显著的嵌套分布格局。 种类-地点矩阵(表3)嵌套程度显著高于NODF软件 随机产生的 1,000 个群落矩阵 $(\mathrm{WNODF}=42.40, P<$

\begin{tabular}{|c|c|c|c|c|c|c|}
\hline $\begin{array}{c}\text { 物种 } \\
\text { Species }\end{array}$ & $\begin{array}{c}\text { 最小需求面积 } \\
\text { Minimum area re- } \\
\text { quirement (ha) }\end{array}$ & $\begin{array}{c}\text { 翅展 } \\
\text { Wingspan } \\
(\mathrm{mm})\end{array}$ & $\begin{array}{c}\text { 体重 } \\
\text { Body weight } \\
(\mathrm{mg})\end{array}$ & $\begin{array}{c}\text { 占据岛屿数 } \\
\text { Number of islands } \\
\text { occupied }\end{array}$ & $\begin{array}{c}\text { 多度 } \\
\text { Abundance }\end{array}$ & $\begin{array}{c}\text { 嵌套序列 } \\
\text { Nested matrix rank }\end{array}$ \\
\hline 酢浆灰蝶 Pseudozizeeria maha & 4.47 & 19.38 & 3.54 & 27 & 1,283 & 1 \\
\hline 红灰蝶 Lycaena phlaeas & 2.55 & 23.46 & 11.04 & 26 & 530 & 2 \\
\hline 黄钩蛱蝶 Polygonia c-aureum & 23.94 & 46.00 & 40.21 & 25 & 749 & 3 \\
\hline 菜粉蝶 Pieris rapae & 82.10 & 38.16 & 15.42 & 23 & 571 & 4 \\
\hline 东方菜粉蝶 Pieris canidia & 24.26 & 42.43 & 17.27 & 22 & 686 & 5 \\
\hline 柑橘凤蝶 Papilio xuthus & 82.10 & 82.45 & 118.44 & 22 & 220 & 6 \\
\hline 玉带凤蝶 Papilio polytes & 82.10 & 81.52 & 130.48 & 21 & 363 & 7 \\
\hline 宽边黄粉蝶 Eurema hecabe & 82.10 & 38.18 & 15.15 & 21 & 290 & 8 \\
\hline 碧凤蝶 Papilio bianor & 82.10 & 98.17 & 188.99 & 20 & 157 & 9 \\
\hline 亮灰蝶 Lampides boeticus & 82.10 & 24.39 & 9.41 & 18 & 124 & 10 \\
\hline 青凤蝶 Graphium sarpedon & 82.10 & 54.73 & 92.09 & 17 & 280 & 11 \\
\hline 点玄灰蝶 Tongeia filicaudis & 23.94 & 17.54 & 3.19 & 15 & 55 & 12 \\
\hline 东亚嚾眼蝶 Ypthima motschulskyi & 82.10 & 30.10 & 10.97 & 14 & 110 & 13 \\
\hline 蓝灰蝶 Everes argiades & 171.70 & 20.33 & 3.88 & 13 & 28 & 14 \\
\hline 黑脉蛱蝶 Hestina assimilis & 109.10 & 70.96 & 144.89 & 12 & 54 & 15 \\
\hline 斐豹蛱蝶 Argyreus hyperbius & 109.10 & 64.92 & 83.06 & 11 & 20 & 16 \\
\hline 曲纹紫灰蝶 Chilades pandava & 82.10 & 21.78 & 7.90 & 10 & 170 & 17 \\
\hline
\end{tabular}

表2 舟山群岛 28 个岛屿蝶类生活史特征参数

Table 2 Life-history traits of butterfly species on 28 islands in the Zhoushan Archipelago 
表2 (续) Table 2 (continued)

\begin{tabular}{|c|c|c|c|c|c|c|}
\hline $\begin{array}{c}\text { 物种 } \\
\text { Species }\end{array}$ & $\begin{array}{c}\text { 最小需求面积 } \\
\text { Minimimum area re- } \\
\text { quirement (ha) }\end{array}$ & $\begin{array}{c}\text { 翅展 } \\
\text { Wingspan } \\
(\mathrm{mm})\end{array}$ & $\begin{array}{c}\text { 体重 } \\
\text { Body weight } \\
\text { (mg) }\end{array}$ & $\begin{array}{c}\text { 占据岛屿数 } \\
\text { Number of islands } \\
\text { occupied }\end{array}$ & $\begin{array}{c}\text { 多度 } \\
\text { Abundance }\end{array}$ & $\begin{array}{c}\text { 嵌套序列 } \\
\text { Nested matrix rank }\end{array}$ \\
\hline 琉璃蛱蝶 Kaniska canace & 109.10 & 54.98 & 113.29 & 10 & 23 & 18 \\
\hline 红珠凤蝶 Pachliopta aristolochiae & 512.40 & 70.08 & 92.31 & 9 & 55 & 19 \\
\hline 直纹稻弄蝶 Parnara guttata & 109.10 & 28.86 & 35.20 & 9 & 47 & 20 \\
\hline 斑缘豆粉蝶 Colias erate & 82.10 & 44.83 & 33.62 & 9 & 25 & 21 \\
\hline 残锷线蛱蝶 Limenitis sulpitia & 222.20 & 53.12 & 53.27 & 8 & 18 & 22 \\
\hline 琉璃灰蝶 Celastrina argiola & 117.90 & 25.29 & 6.63 & 8 & 15 & 23 \\
\hline 蒙链荫眼蝶 Neope muirheadii & 377.80 & 58.16 & 79.98 & 8 & 14 & 24 \\
\hline 南亚谷弄蝶 Pelopidas agna & $1,109.80$ & 31.35 & 40.00 & 7 & 19 & 25 \\
\hline 白带螯蛱蝶 Charaxes bernardus & 109.10 & 62.89 & 290.54 & 7 & 12 & 26 \\
\hline 稻眉眼蝶 Mycalesis gotama & 82.10 & 34.05 & 15.55 & 7 & 11 & 27 \\
\hline 丝带凤蝶 Sericinus montelus & $1,092.00$ & 55.12 & 34.28 & 6 & 80 & 28 \\
\hline 大红蛱蝶 Vanessa indica & 117.90 & 51.49 & 79.06 & 6 & 16 & 29 \\
\hline 隐纹谷弄蝶 Pelopidas mathias & 171.70 & 28.70 & 32.18 & 6 & 8 & 30 \\
\hline 曲纹黄室弄蝶 Potanthus flavus & 222.20 & 24.90 & 28.50 & 6 & 8 & 31 \\
\hline 幻紫斑蛱蝶 Hypolimnas bolina & $1,700.50$ & 63.76 & 98.92 & 5 & 20 & 32 \\
\hline 大紫琉璃灰蝶 Celastrina oreas & 109.10 & 24.65 & 8.42 & 5 & 17 & 33 \\
\hline 蓝凤蝶 Papilio protenor & 143.90 & 101.47 & 172.24 & 5 & 15 & 34 \\
\hline 小环蛱蝶 Neptis sappho & 303.90 & 45.57 & 24.40 & 5 & 10 & 35 \\
\hline 二尾蛱蝶 Polyura narcaea & $2,135.00$ & 63.45 & 171.16 & 5 & 10 & 36 \\
\hline 青豹蛱蝶 Damora sagana & 109.10 & 64.16 & 185.42 & 5 & 6 & 37 \\
\hline 小红蛱蝶 Vanessa cardui & 117.90 & 42.26 & 49.73 & 5 & 6 & 38 \\
\hline 金凤蝶 Papilio machaon & $1,092.00$ & 73.52 & 96.51 & 4 & 43 & 39 \\
\hline 黄斑弄蝶 Ampittia dioscorides & $5,979.20$ & 19.28 & 9.05 & 4 & 26 & 40 \\
\hline 高沙子燕灰蝶 Rapala takasagonis & $2,690.20$ & 26.86 & 17.24 & 4 & 10 & 41 \\
\hline 放踵珂弄蝶 Caltoris cahira & 422.40 & 29.86 & 25.90 & 4 & 7 & 42 \\
\hline 柳紫闪蛱蝶 Apatura ilia & 377.80 & 55.87 & 112.87 & 4 & 7 & 43 \\
\hline 尖翅银灰蝶 Curetis acuta & $2,837.40$ & 36.29 & 39.74 & 4 & 5 & 44 \\
\hline 蛇眼蝶 Minois dryas & 422.40 & 49.67 & 52.20 & 3 & 10 & 45 \\
\hline 铸凤蝶 Byasa alcinous & $1,092.00$ & 74.64 & 101.40 & 3 & 8 & 46 \\
\hline 美凤蝶 Papilio memnon & 222.20 & 106.41 & 168.60 & 3 & 3 & 47 \\
\hline 小眉眼蝶 Mycalesis mineus & $4,037.00$ & 32.81 & 16.38 & 2 & 17 & 48 \\
\hline 美眼蛱蝶 Junonia almana & $7,735.20$ & 45.11 & 51.49 & 2 & 16 & 49 \\
\hline 方斑珂弄蝶 Caltoris cormasa & $2,690.20$ & 35.05 & 56.65 & 2 & 2 & 50 \\
\hline 红翅长标弄蝶 Telicota ancilla & $9,365.80$ & 21.65 & 23.45 & 2 & 2 & 51 \\
\hline 忘忧尾蛱蝶 Polyura nepenthes & $2,690.20$ & 85.01 & 242.70 & 2 & 2 & 52 \\
\hline 幺纹稻弄蝶 Parnara baba & $7,735.20$ & 24.45 & 21.61 & 1 & 8 & 53 \\
\hline 豹弄蝶 Thymelicus leoninus & 358.60 & 22.69 & 9.40 & 1 & 4 & 54 \\
\hline 金斑蝶 Danaus chrysippus & $4,037.00$ & 53.99 & 63.30 & 1 & 2 & 55 \\
\hline 小黄斑弄蝶 Ampittia nana & $47,616.50$ & 11.99 & 3.85 & 1 & 2 & 56 \\
\hline 长纹黛眼蝶 Lethe europa & $47,616.50$ & 48.49 & 51.40 & 1 & 1 & 57 \\
\hline 罣眼蝶 Ypthima balda & $2,690.20$ & 28.08 & 12.90 & 1 & 1 & 58 \\
\hline 虎斑蝶 Danaus genutia & $9,365.80$ & 76.80 & 128.40 & 1 & 1 & 59 \\
\hline 戟眉线蛱蝶 Limenitis homeyeri & 377.80 & 51.85 & 33.60 & 1 & 1 & 60 \\
\hline 链环蛱蝶 Neptis pryeri & $2,690.20$ & 48.87 & 19.80 & 1 & 1 & 61 \\
\hline 曲纹蜘蛱蝶 Araschnia doris & $2,690.20$ & 35.71 & 13.80 & 1 & 1 & 62 \\
\hline 玉斑凤蝶 Papilio helenus & $47,616.50$ & 103.49 & 197.70 & 1 & 1 & 63 \\
\hline 优秀酒灰蝶 Satyrium eximium & 358.60 & 26.96 & 21.80 & 1 & 1 & 64 \\
\hline
\end{tabular}


表3 舟山群岛 28 个岛屿蝶类种类-地点最大化排序嵌套矩阵

Table 3 Maximally ranked species-habitat nested matrix for butterfly on 28 islands in the Zhoushan Archipelago

\begin{tabular}{|c|c|c|c|c|c|c|c|c|c|c|c|c|c|c|c|c|c|c|c|c|c|c|c|c|c|c|c|c|}
\hline \multirow[t]{2}{*}{ 物种 Species } & \multicolumn{28}{|c|}{ 岛屿编号 Island code } \\
\hline & 1 & 3 & 2 & 7 & 4 & 9 & 8 & 6 & 5 & 10 & 12 & 23 & 16 & 15 & 17 & 20 & 19 & 24 & 18 & 14 & 11 & 13 & 21 & 22 & 26 & 25 & 27 & 28 \\
\hline 酢浆灰蝶 Pseudozizeeria maha & 1 & 1 & 1 & 1 & 1 & 1 & 1 & 1 & 1 & 1 & 1 & 1 & 1 & 1 & 1 & 1 & 1 & 1 & 1 & 1 & 1 & 1 & 1 & 1 & 1 & 1 & 1 & 0 \\
\hline 红灰蝶 Lycaena phlaeas & 1 & 1 & 1 & 1 & 1 & 1 & 1 & 1 & 1 & 1 & 1 & 1 & 1 & 1 & 1 & 1 & 0 & 1 & 1 & 1 & 1 & 1 & 0 & 1 & 1 & 1 & 1 & 1 \\
\hline 黄钩蛱蝶 Polygonia c-aureum & 1 & 1 & 1 & 1 & 1 & 1 & 0 & 1 & 1 & 1 & 1 & 1 & 1 & 1 & 1 & 1 & 1 & 1 & 1 & 1 & 1 & 1 & 1 & 1 & 1 & 1 & 0 & 0 \\
\hline 菜粉蝶 Pieris rapae & 1 & 1 & 1 & 1 & 1 & 1 & 1 & 1 & 1 & 1 & 1 & 1 & 1 & 1 & 1 & 1 & 0 & 1 & 1 & 1 & 1 & 1 & 1 & 1 & 0 & 0 & 0 & 0 \\
\hline 东方菜粉蝶 Pieris canidia & 1 & 1 & 1 & 1 & 1 & 1 & 1 & 1 & 1 & 1 & 1 & 0 & 1 & 1 & 1 & 1 & 1 & 1 & 1 & 1 & 1 & 0 & 0 & 1 & 0 & 1 & 0 & 0 \\
\hline 柑橘凤蝶 Papilio xuthus & 1 & 1 & 1 & 1 & 1 & 1 & 1 & 1 & 1 & 1 & 1 & 1 & 1 & 1 & 1 & 1 & 0 & 1 & 0 & 1 & 1 & 1 & 1 & 1 & 0 & 0 & 0 & 0 \\
\hline 玉带凤蝶 Papilio polytes & 1 & 1 & 1 & 1 & 1 & 1 & 1 & 1 & 1 & 1 & 1 & 1 & 1 & 0 & 0 & 1 & 1 & 1 & 1 & 1 & 1 & 1 & 1 & 0 & 0 & 0 & 0 & 0 \\
\hline 宽边黄粉蝶 Eurema hecabe & 1 & 1 & 1 & 1 & 1 & 1 & 1 & 1 & 1 & 1 & 1 & 1 & 1 & 1 & 1 & 1 & 1 & 1 & 1 & 0 & 1 & 0 & 1 & 0 & 0 & 0 & 0 & 0 \\
\hline 碧凤蝶 Papilio bianor & 1 & 1 & 1 & 1 & 1 & 1 & 1 & 1 & 1 & 1 & 0 & 1 & 1 & 1 & 1 & 1 & 1 & 1 & 1 & 0 & 1 & 0 & 1 & 0 & 0 & 0 & 0 & 0 \\
\hline 亮灰蝶 Lampides boeticus & 1 & 1 & 1 & 1 & 1 & 1 & 1 & 0 & 1 & 1 & 0 & 1 & 1 & 1 & 1 & 1 & 1 & 1 & 0 & 1 & 1 & 0 & 0 & 0 & 0 & 0 & 0 & 0 \\
\hline 青风蝶 Graphium sarpedon & 1 & 1 & 1 & 1 & 1 & 1 & 1 & 1 & 1 & 0 & 1 & 1 & 1 & 0 & 0 & 0 & 1 & 1 & 1 & 0 & 0 & 1 & 1 & 0 & 0 & 0 & 0 & 0 \\
\hline 点玄灰蝶 Tongeia filicaudis & 0 & 1 & 0 & 1 & 0 & 1 & 1 & 1 & 0 & 1 & 1 & 1 & 1 & 1 & 0 & 1 & 0 & 1 & 0 & 1 & 0 & 0 & 0 & 1 & 1 & 0 & 0 & 0 \\
\hline 东亚翟眼蝶 Ypthima motschulskyi & 1 & 1 & 0 & 1 & 1 & 1 & 1 & 0 & 1 & 0 & 1 & 1 & 1 & 0 & 0 & 0 & 1 & 1 & 0 & 0 & 1 & 0 & 1 & 0 & 0 & 0 & 0 & 0 \\
\hline 蓝灰蝶 Everes argiades & 1 & 1 & 1 & 1 & 0 & 1 & 0 & 1 & 1 & 1 & 1 & 0 & 0 & 1 & 1 & 1 & 1 & 0 & 0 & 0 & 0 & 0 & 0 & 0 & 0 & 0 & 0 & 0 \\
\hline 黑脉蛱蝶 Hestina assimilis & 1 & 1 & 1 & 0 & 0 & 1 & 1 & 1 & 0 & 1 & 0 & 1 & 0 & 0 & 0 & 1 & 1 & 0 & 1 & 1 & 0 & 0 & 0 & 0 & 0 & 0 & 0 & 0 \\
\hline 斐豹蛱蝶 Argyreus hyperbius & 1 & 1 & 1 & 1 & 0 & 0 & 1 & 0 & 1 & 1 & 0 & 1 & 0 & 1 & 0 & 0 & 0 & 0 & 1 & 0 & 0 & 0 & 1 & 0 & 0 & 0 & 0 & 0 \\
\hline 曲纹紫灰蝶 Chilades pandava & 1 & 1 & 0 & 1 & 1 & 1 & 1 & 0 & 0 & 0 & 0 & 0 & 1 & 0 & 0 & 0 & 1 & 1 & 0 & 0 & 1 & 0 & 0 & 0 & 0 & 0 & 0 & 0 \\
\hline 琉璃蛱蝶 Kaniska canace & 1 & 1 & 1 & 1 & 1 & 0 & 1 & 1 & 1 & 0 & 0 & 1 & 0 & 0 & 1 & 0 & 0 & 0 & 0 & 0 & 0 & 0 & 0 & 0 & 0 & 0 & 0 & 0 \\
\hline 红珠凤蝶 Pachliopta aristolochiae & e 1 & 1 & 1 & 0 & 1 & 0 & 0 & 1 & 0 & 1 & 1 & 0 & 0 & 0 & 0 & 0 & 0 & 0 & 0 & 1 & 0 & 1 & 0 & 0 & 0 & 0 & 0 & 0 \\
\hline 直纹稻弄蝶 Parnara guttata & 1 & 0 & 1 & 1 & 1 & 0 & 0 & 1 & 0 & 0 & 0 & 1 & 0 & 1 & 1 & 1 & 0 & 0 & 0 & 0 & 0 & 0 & 0 & 0 & 0 & 0 & 0 & 0 \\
\hline 斑缘豆粉蝶 Colias erate & 1 & 1 & 1 & 1 & 0 & 1 & 0 & 0 & 1 & 0 & 0 & 0 & 0 & 0 & 0 & 0 & 0 & 1 & 1 & 0 & 0 & 1 & 0 & 0 & 0 & 0 & 0 & 0 \\
\hline 残锷线蛱蝶 Limenitis sulpitia & 0 & 1 & 1 & 1 & 1 & 1 & 1 & 0 & 0 & 0 & 0 & 0 & 1 & 0 & 0 & 0 & 1 & 0 & 0 & 0 & 0 & 0 & 0 & 0 & 0 & 0 & 0 & 0 \\
\hline 琉璃灰蝶 Celastrin & 1 & 1 & 0 & 1 & 1 & 0 & 0 & 1 & 0 & 1 & 0 & 0 & 1 & 0 & 0 & 0 & 0 & 0 & 0 & 0 & 0 & 0 & 0 & 1 & 0 & 0 & 0 & 0 \\
\hline 蒙链荫眼蝶 Neope muirheadii & 1 & 0 & 1 & 1 & 1 & 1 & 1 & 0 & 1 & 0 & 0 & 0 & 1 & 0 & 0 & 0 & 0 & 0 & 0 & 0 & 0 & 0 & 0 & 0 & 0 & 0 & 0 & 0 \\
\hline 南亚谷弄蝶 Pelopidas agna & 1 & 0 & 1 & 1 & 1 & 1 & 0 & 0 & 1 & 0 & 1 & 0 & 0 & 0 & 0 & 0 & 0 & 0 & 0 & 0 & 0 & 0 & 0 & 0 & 0 & 0 & 0 & 0 \\
\hline 白带螯蛱蝶 Chara & 0 & 1 & 0 & 0 & 1 & 1 & 1 & 0 & 0 & 0 & 1 & 1 & 0 & 0 & 0 & 0 & 0 & 0 & 1 & 0 & 0 & 0 & 0 & 0 & 0 & 0 & 0 & 0 \\
\hline 稻眉眼蝶 Mycalesis gotama & 1 & 1 & 0 & 0 & 1 & 1 & 0 & 1 & 0 & 0 & 0 & 0 & 0 & 0 & 0 & 0 & 0 & 1 & 1 & 0 & 0 & 0 & 0 & 0 & 0 & 0 & 0 & 0 \\
\hline 丝带凤蝶 Sericinus montelus & 1 & 1 & 1 & 0 & 1 & 0 & 0 & 0 & 0 & 0 & 1 & 0 & 0 & 0 & 0 & 0 & 0 & 0 & 0 & 0 & 0 & 1 & 0 & 0 & 0 & 0 & 0 & 0 \\
\hline 大红蛱蝶 Vanessa $i$ & 1 & 1 & 0 & 0 & 0 & 0 & 0 & 0 & 0 & 1 & 0 & 0 & 0 & 1 & 1 & 0 & 0 & 0 & 0 & 0 & 0 & 0 & 0 & 1 & 0 & 0 & 0 & 0 \\
\hline 隐纹谷弄蝶 Pelopidas mathias & 0 & 1 & 1 & 1 & 1 & 0 & 0 & 0 & 1 & 0 & 0 & 0 & 0 & 0 & 0 & 1 & 0 & 0 & 0 & 0 & 0 & 0 & 0 & 0 & 0 & 0 & 0 & 0 \\
\hline 曲纹黄室弄蝶 Potanthus flavus & 1 & 1 & 1 & 0 & 0 & 0 & 0 & 0 & 0 & 0 & 1 & 0 & 0 & 0 & 0 & 0 & 1 & 0 & 0 & 1 & 0 & 0 & 0 & 0 & 0 & 0 & 0 & 0 \\
\hline 幻紫斑蛱蝶 Hypolim & 0 & 1 & 0 & 1 & 0 & 0 & 0 & 1 & 1 & 0 & 0 & 0 & 0 & 0 & 0 & 0 & 0 & 0 & 0 & 0 & 1 & 0 & 0 & 0 & 0 & 0 & 0 & 0 \\
\hline 大紫琉璃灰蝶 Celastrina & 1 & 0 & 0 & 0 & 0 & 0 & 0 & 1 & 0 & 1 & 0 & 1 & 0 & 0 & 0 & 0 & 0 & 0 & 1 & 0 & 0 & 0 & 0 & 0 & 0 & 0 & 0 & 0 \\
\hline 蓝凤蝶 Papilio protenor & 1 & 1 & 0 & 1 & 0 & 0 & 0 & 0 & 1 & 0 & 0 & 0 & 0 & 0 & 0 & 0 & 0 & 0 & 0 & 0 & 0 & 0 & 1 & 0 & 0 & 0 & 0 & 0 \\
\hline 小环蛱蝶 Neptis sappho & 1 & 1 & 0 & 0 & 0 & 1 & 0 & 0 & 0 & 0 & 1 & 0 & 0 & 0 & 0 & 0 & 0 & 0 & 1 & 0 & 0 & 0 & 0 & 0 & 0 & 0 & 0 & 0 \\
\hline 二尾蛱蝶 Polyura narcaea & 1 & 0 & 1 & 0 & 0 & 0 & 1 & 1 & 0 & 1 & 0 & 0 & 0 & 0 & 0 & 0 & 0 & 0 & 0 & 0 & 0 & 0 & 0 & 0 & 0 & 0 & 0 & 0 \\
\hline 青豹蛱蝶 Damora sagana & 1 & 0 & 1 & 1 & 0 & 0 & 0 & 0 & 0 & 0 & 0 & 1 & 0 & 0 & 0 & 0 & 1 & 0 & 0 & 0 & 0 & 0 & 0 & 0 & 0 & 0 & 0 & 0 \\
\hline 小红蛱蝶 Vanessa cardui & 0 & 0 & 0 & 0 & 0 & 0 & 0 & 0 & 0 & 1 & 0 & 0 & 0 & 1 & 1 & 1 & 0 & 0 & 0 & 0 & 0 & 0 & 0 & 1 & 0 & 0 & 0 & 0 \\
\hline 金凤蝶 Papilio machaon & 0 & 0 & 1 & 0 & 0 & 0 & 1 & 0 & 0 & 0 & 1 & 0 & 0 & 0 & 0 & 0 & 0 & 0 & 0 & 0 & 0 & 1 & 0 & 0 & 0 & 0 & 0 & 0 \\
\hline 黄斑弄蝶 Ampittia dioscorides & 1 & 0 & 1 & 0 & 1 & 0 & 0 & 1 & 0 & 0 & 0 & 0 & 0 & 0 & 0 & 0 & 0 & 0 & 0 & 0 & 0 & 0 & 0 & 0 & 0 & 0 & 0 & 0 \\
\hline 高沙子燕灰蝶 Rapala takasagonis & $s 1$ & 1 & 0 & 1 & 0 & 1 & 0 & 0 & 0 & 0 & 0 & 0 & 0 & 0 & 0 & 0 & 0 & 0 & 0 & 0 & 0 & 0 & 0 & 0 & 0 & 0 & 0 & 0 \\
\hline 放踵珂弄蝶 Caltoris cahira & 1 & 1 & 0 & 1 & 0 & 0 & 0 & 0 & 0 & 0 & 0 & 0 & 0 & 1 & 0 & 0 & 0 & 0 & 0 & 0 & 0 & 0 & 0 & 0 & 0 & 0 & 0 & 0 \\
\hline 柳紫闪蛱蝶 Apatura ilia & 0 & 1 & 1 & 0 & 1 & 0 & 0 & 0 & 0 & 0 & 0 & 0 & 1 & 0 & 0 & 0 & 0 & 0 & 0 & 0 & 0 & 0 & 0 & 0 & 0 & 0 & 0 & 0 \\
\hline 尖翅银灰蝶 Curetis acuta & 1 & 1 & 0 & 0 & 1 & 0 & 1 & 0 & 0 & 0 & 0 & 0 & 0 & 0 & 0 & 0 & 0 & 0 & 0 & 0 & 0 & 0 & 0 & 0 & 0 & 0 & 0 & 0 \\
\hline 蛇眼蝶 Minois dryas & 0 & 0 & 1 & 0 & 0 & 0 & 0 & 0 & 0 & 0 & 0 & 0 & 0 & 1 & 0 & 0 & 0 & 0 & 0 & 1 & 0 & 0 & 0 & 0 & 0 & 0 & 0 & 0 \\
\hline 锦风蝶 Byasa alcinous & 0 & 0 & 0 & 1 & 0 & 0 & 0 & 0 & 0 & 0 & 1 & 0 & 0 & 0 & 0 & 0 & 0 & 0 & 0 & 0 & 0 & 1 & 0 & 0 & 0 & 0 & 0 & 0 \\
\hline 美凤蝶 Papilio memnon & 0 & 1 & 1 & 0 & 0 & 0 & 0 & 0 & 0 & 0 & 0 & 0 & 0 & 0 & 0 & 0 & 1 & 0 & 0 & 0 & 0 & 0 & 0 & 0 & 0 & 0 & 0 & 0 \\
\hline 小眉眼蝶 Mycalesis mineus & 1 & 0 & 0 & 1 & 0 & 0 & 0 & 0 & 0 & 0 & 0 & 0 & 0 & 0 & 0 & 0 & 0 & 0 & 0 & 0 & 0 & 0 & 0 & 0 & 0 & 0 & 0 & 0 \\
\hline 美眼蛱蝶 Junonia almana & 1 & 0 & 0 & 0 & 1 & 0 & 0 & 0 & 0 & 0 & 0 & 0 & 0 & 0 & 0 & 0 & 0 & 0 & 0 & 0 & 0 & 0 & 0 & 0 & 0 & 0 & 0 & 0 \\
\hline 方斑珂弄蝶 Caltoris cormasa & 0 & 1 & 0 & 0 & 0 & 1 & 0 & 0 & 0 & 0 & 0 & 0 & 0 & 0 & 0 & 0 & 0 & 0 & 0 & 0 & 0 & 0 & 0 & 0 & 0 & 0 & 0 & 0 \\
\hline 红翅长标弄蝶 Telicota ancilla & 0 & 1 & 1 & 0 & 0 & 0 & 0 & 0 & 0 & 0 & 0 & 0 & 0 & 0 & 0 & 0 & 0 & 0 & 0 & 0 & 0 & 0 & 0 & 0 & 0 & 0 & 0 & 0 \\
\hline 忘忧尾蛱蝶 Polyura nepenthes & 0 & 0 & 0 & 0 & 0 & 1 & 1 & 0 & 0 & 0 & 0 & 0 & 0 & 0 & 0 & 0 & 0 & 0 & 0 & 0 & 0 & 0 & 0 & 0 & 0 & 0 & 0 & 0 \\
\hline 么纹稻弄蝶 Parnara baba & 0 & 0 & 0 & 0 & 1 & 0 & 0 & 0 & 0 & 0 & 0 & 0 & 0 & 0 & 0 & 0 & 0 & 0 & 0 & 0 & 0 & 0 & 0 & 0 & 0 & 0 & 0 & 0 \\
\hline
\end{tabular}


表3 (续) Table 3 (continued)

物种 Species 岛屿编号 Island code*

豹弄蝶 Thymelicus leoninus

$\begin{array}{llllllllllllllllllllllllllllll}0 & 0 & 0 & 0 & 0 & 0 & 0 & 0 & 0 & 0 & 0 & 0 & 0 & 0 & 1 & 0 & 0 & 0 & 0 & 0 & 0 & 0 & 0 & 0 & 0 & 0 & 0 & 0\end{array}$

金斑蝶 Danaus chrysippus

小黄斑弄蝶 Ampittia nana

$\begin{array}{llllllllllllllllllllllllllll}0 & 0 & 0 & 1 & 0 & 0 & 0 & 0 & 0 & 0 & 0 & 0 & 0 & 0 & 0 & 0 & 0 & 0 & 0 & 0 & 0 & 0 & 0 & 0 & 0 & 0 & 0 & 0\end{array}$

长纹黛眼蝶 Lethe europa

$\begin{array}{llllllllllllllllllllllllllll}1 & 0 & 0 & 0 & 0 & 0 & 0 & 0 & 0 & 0 & 0 & 0 & 0 & 0 & 0 & 0 & 0 & 0 & 0 & 0 & 0 & 0 & 0 & 0 & 0 & 0 & 0 & 0\end{array}$

篗眼蝶 Ypthima balda

$\begin{array}{llllllllllllllllllllllllllll}1 & 0 & 0 & 0 & 0 & 0 & 0 & 0 & 0 & 0 & 0 & 0 & 0 & 0 & 0 & 0 & 0 & 0 & 0 & 0 & 0 & 0 & 0 & 0 & 0 & 0 & 0 & 0\end{array}$

虎斑蝶 Danaus genutia

$\begin{array}{llllllllllllllllllllllllllll}0 & 0 & 0 & 0 & 0 & 1 & 0 & 0 & 0 & 0 & 0 & 0 & 0 & 0 & 0 & 0 & 0 & 0 & 0 & 0 & 0 & 0 & 0 & 0 & 0 & 0 & 0 & 0\end{array}$

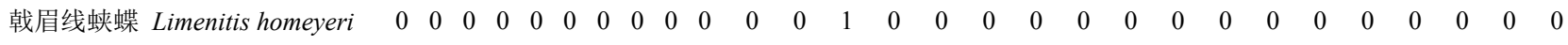

$\begin{array}{llllllllllllllllllllllllllllll}\text { 链环蛱蝶 Neptis pryeri } & 0 & 0 & 0 & 0 & 0 & 1 & 0 & 0 & 0 & 0 & 0 & 0 & 0 & 0 & 0 & 0 & 0 & 0 & 0 & 0 & 0 & 0 & 0 & 0 & 0 & 0 & 0 & 0\end{array}$

曲纹蜘蛱蝶 Araschnia doris $\quad \begin{array}{llllllllllllllllllllllllllllllll}0 & 0 & 0 & 0 & 0 & 1 & 0 & 0 & 0 & 0 & 0 & 0 & 0 & 0 & 0 & 0 & 0 & 0 & 0 & 0 & 0 & 0 & 0 & 0 & 0 & 0 & 0 & 0\end{array}$

$\begin{array}{lllllllllllllllllllllllllllllll}\text { 玉斑风蝶 Papilio helenus } & 1 & 0 & 0 & 0 & 0 & 0 & 0 & 0 & 0 & 0 & 0 & 0 & 0 & 0 & 0 & 0 & 0 & 0 & 0 & 0 & 0 & 0 & 0 & 0 & 0 & 0 & 0 & 0\end{array}$

优秀酒灰蝶 Satyrium eximium $\quad 0$\begin{tabular}{llllllllllllllllllllllllllll} 
& 0 & 0 & 0 & 0 & 0 & 0 & 0 & 0 & 0 & 0 & 0 & 0 & 0 & 1 & 0 & 0 & 0 & 0 & 0 & 0 & 0 & 0 & 0 & 0 & 0 & 0 & 0 \\
\hline
\end{tabular}

岛屿编号见表1。The codes for all islands are shown in Table 1 .

表4 舟山群岛28个岛屿蝶类群落嵌套程度WNODF量化及嵌套影响因素partial Spearman秩相关分析结果

Table 4 Results of nestedness quantified by WNODF and partial Spearman rank correlations of influences on nestedness for butterfly assemblages on 28 islands in the Zhoushan Archipelago

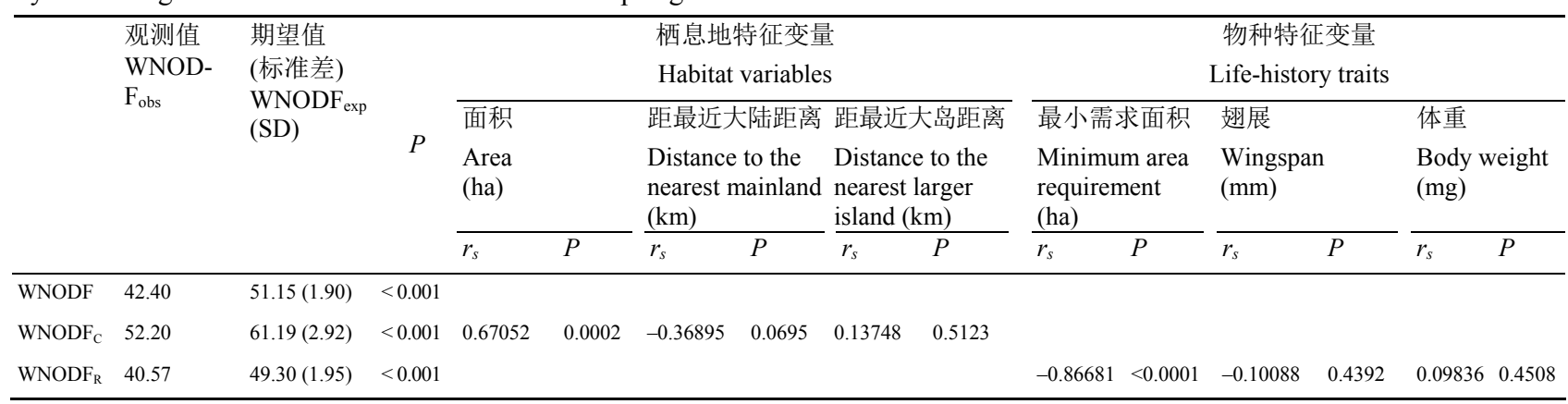

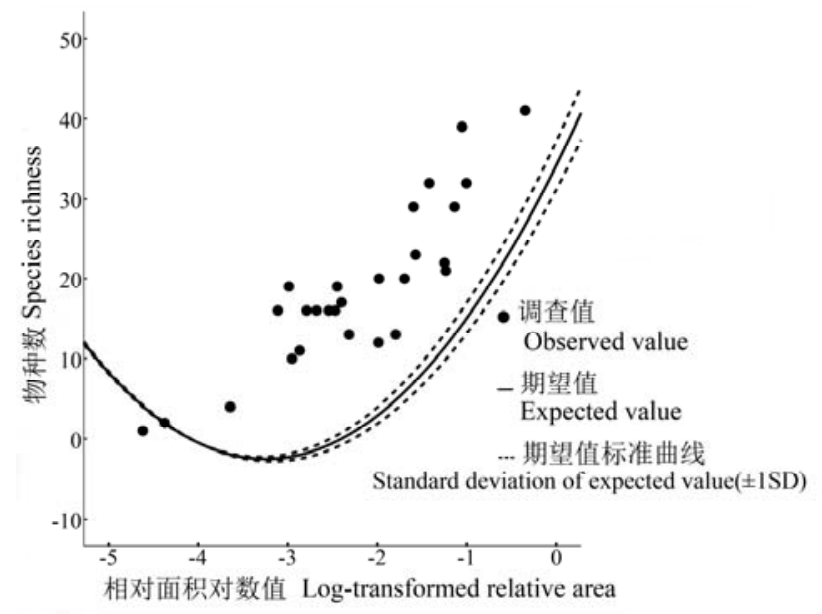

图 2 舟山群岛 28 个取样岛屿蝶类物种数调查值与随机分布 模型预测结果比较

Fig. 2 Comparison of observed data to expected values under the random placement model for butterfly in the Zhoushan Archipelago
$0.001)$ (表4)。物种 $\left(\mathrm{WNODF}_{\mathrm{R}}=40.57, P=0.001\right)$ 和研 究岛屿 $\left(\mathrm{WNODF}_{\mathrm{C}}=52.20, P<0.001\right)$ 也分别呈现显 著的嵌套结构。

\section{4 嵌套因素分析结果}

Partial Spearman秩相关分析结果显示, 栖息地 特征变量中岛屿面积与嵌套序列显著正相关 $(r=$ $0.67052, P=0.0002)$, 而反映隔离度的距最近大陆 距离和距最近大岛距离两项参数未显示出显著的 相关性(距最近大陆距离: $r=-0.36895, P=0.0695$; 距最近大岛距离: $r=0.13748, P=0.5123) 。$ 物种生 活史特征参数中仅蝶类最小需求面积与嵌套序列 有显著的负相关性 $(r=-0.86681, P<0.0001)$, 翅展 和体重两项参数与嵌套序列相关性不显著 $($ 翅展: $r=$ $-0.10088, P=0.4392$; 体重: $r=0.09836, P=$ $0.4508)$ 。

在根据随机分布模型计算所得的各岛物种数 期望值与岛屿相对面积对数值构建的预测种一面积 
关系曲线中, 仅 1 个调查点在预测种-面积关系曲线 标准差之内(图2), 此结果表明舟山群岛蝶类群落分 布不是随机的。

\section{4 讨论}

目前, 嵌套分布格局的研究主要集中于大陆片 断化森林斑块和湖泊岛屿中的脊椎动物(Menezes \& Fernandez, 2013; Rodríguez \& Ojeda, 2013; Tabak et al, 2014)。海洋性群岛形成时间长, 介质均为海水, 各岛物种分布稳定, 是研究栖息地片断化理论及物 种嵌套分布格局的自然实验系统。然而, 除脊椎动 物类群如两栖类外(Li et al, 1998), 目前对海洋性群 岛中无脊椎动物群落组成和结构进行深入嵌套分 析的研究尚不多见。

我们的研究表明, 舟山群岛蝶类群落分布呈现 显著的嵌套结构。在影响蝶类群落分布的栖息地特 征和生活史特征变量中, 反映蝶类灭绝倾向的岛屿 面积和最小需求面积两项参数与嵌套序列显著相 关, 而反映物种扩散能力的隔离度和翅展与嵌套序 列无显著相关性, 由此可知, 舟山群岛蝶类群落嵌 套结构支持选择性灭绝假说, 而否定选择性迁入假 说。另外, 随机模型结果显示舟山群岛蝶类群落分 布是非随机的, 这说明被动抽样也不是该群落嵌套 结构形成的原因。

我们认为舟山群岛蝶类群落嵌套结构的形成 机制符合选择性灭绝假说: 岛屿面积和物种最小需 求面积反映物种灭绝趋势的变量与嵌套序列有显 著相关性。选择性灭绝被认为是正在经历物种丧失 和“松驰”的片断化生境中物种群落嵌套格局形成的 主导因素(Driscoll, 2008; Hill et al, 2011)。物种的“松 弛” 速率很大程度上取决于斑块面积、隔离度等因 素(Ferraz et al, 2003)。斑块面积在一定程度上反映 了可供物种利用的资源量和空间分布范围, 在我们 的研究中, 那些最小需求面积大的物种灭绝风险相 对较高, 会先于其他物种从岛上消失, 从而形成嵌 套结构(Li et al, 1998; Wright et al, 1998; Wang et al, 2010, 2012)。

因为反映物种扩散能力的翅展与嵌套序列没 有显著相关性, 所以选择性迁入假说可能无法解释 舟山群岛蝶类群落嵌套结构的形成。Soga和Koike (2012)规定选取的研究斑块间的最小距离必须大于 $800 \mathrm{~m}$, 以避免假重复, 但已有研究表明某些蝶类
在海洋性陆桥岛屿间可进行上千公里距离的迁徙 (Dover \& Settele, 2009), 因而, 舟山群岛海岛间的 隔离度并不能阻止蝶类发生迁移。另外，小岛屿在 物种扩散过程中可起到“踏脚石”的作用(Uezu et al, 2008; Soga \& Koike, 2013), 也可能是导致岛屿间隔 离度对舟山群岛蝶类群落嵌套结构影响较小的原 因。

被动抽样假说强调嵌套格局形成的方法因素, 在实际研究中应当尽量避免取样效应的干扰 (Morrison, 2013)。Andrén等强调在进行嵌套分析时 应首先对被动抽样假说进行检验, 但由于物种多度 数据获取具有一定的难度, 因此取样效应很少获得 检验(Andrén, 1994; Wright et al, 1998), 我们的研究 结果表明被动抽样假说不是舟山群岛蝶类嵌套结 构的成因, 这与Worthen等(1998)和 Wang 等(2010, 2012)的研究结果一致。

综上所述, 舟山群岛蝶类群落的嵌套格局同时 受岛屿景观特征和物种生活史特征两方面的影响。 栖息地特征和物种生活史特征对生物群落结构起 到双重的篮分作用(classification), 共同决定着斑块 中物种的组成, 适应能力强的物种能广泛分布于各 种环境中, 而适应能力弱的物种则局限在相对“宽 松” 的环境中, 进而从整体上产生一种嵌套分布格 局(Wright et al, 1998; Schouten et al, 2007)。我们的 研究结果表明, 那些面积较大的岛屿由于能够为蝶 类提供充足的生存空间和较多的可利用资源, 可能 会包含较多的物种; 同时, 那些对面积需求大的种 类因为对环境高度敏感, 在岛屿上的灭绝风险也较 高。因此, 我们建议, 在制定片断化栖息地中蝶类 物种多样性的保护策略时, 应当充分关注那些分布 在面积较大岛屿和最小需求面积较大的物种。

致谢: 感谢浙江大学丁平教授、王彦平博士、吴玲 兵博士、陈传武博士对实验设计和数据统计的指导; 感谢涂州学院诸立新博士帮助鉴定了部分蝶类物 种; 感谢中国计量大学王嵬栋、季珡、装枳博、李 坤伦和廖海江同学在野外标本采集中的帮助; 感谢 舟山各岛船工为野外工作提供的巨大便利。

\section{参考文献}

Almeida-Neto M, Ulrich W (2011) A straightforward computational approach for measuring nestedness using quantitative matrices. Environment Modelling \& Software, 26, 173-178. 
Andrén H (1994) Can one use nested subset pattern to reject the random sample hypothesis? Examples from boreal bird communities. Oikos, 70, 489-491.

Azeria ET, Kolasa J (2008) Nestedness, niche metrics and temporal dynamics of a metacommunity in a dynamic natural model system. Oikos, 117, 1006-1019.

Benedick S, Hill JK, Mustaffa N, Chey VK, Maryati M, Searle JB, Schilthuizen M, Hamer KC (2006) Impacts of rain forest fragmentation on butterflies in northern Borneo: species richness, turnover and the value of small fragments. Journal of Applied Ecology, 43, 967-977.

Chen SH, Wang YJ (2004) Nestedness pattern of insular community assemblages and its applications. Chinese Journal of Ecology, 23, 81-87. (in Chinese with English abstract) [陈 水华, 王玉军 (2004) 岛屿群落组成的嵌套格局及其应 用. 生态学杂志, 23, 81-87.]

Coleman BD, Mares MA, Willig MR, Hsieh Y-H (1982) Randomness, area, and species richness. Ecology, 63, 11211133.

Darlington PJ (1957) Zoogeography: The Geographical Distribution of Animals. John Wiley \& Sons, New York.

Dennis RLH, Hardy PB, Dapporto L (2012) Nestedness in island faunas: novel insights into island biogeography through butterfly community profiles of colonization ability and migration capacity. Journal of Biogeography, 39, 1412-1426.

Dover J, Settele J (2009) The influences of landscape structure on butterfly distribution and movement: a review. Journal of Insect Conservation, 13, 3-27.

Driscoll DA (2008) The frequency of metapopulations, metacommunities and nestedness in a fragmented landscape. Oikos, 117, 297-309.

Ferraz G, Russell GJ, Stouffer PC, Richard O, Bierregaard J, Pimm SL, Lovejoy TE (2003) Rates of species loss from Amazonian forest fragments. Proceedings of the National Academy of Sciences, USA, 100, 14069-14073.

Florencio M, Díaz-Paniagua C, Serrano L, Bilton DT (2011) Spatio-temporal nested patterns in macroinvertebrate assemblages across a pond network with a wide hydroperiod range. Oecologia, 166, 469-483.

Frick WF, Hayes JP, Heady PA III (2009) Nestedness of desert bat assemblages: species composition patterns in insular and terrestrial landscapes. Oecologia, 158, 687-697.

Gibson L, Lynam AJ, Bradshaw CJA, He F, Bickford DP, Woodruff DS, Bumrungsri S, Laurance WF (2013) Nearcomplete extinction of native small mammal fauna 25 years after forest fragmentation. Science, 341, 1508-1510.

Habel JC, Ulrich W, Assmann T, Masters J (2013) Allele elimination recalculated: nested subset analyses for molecular biogeographical data. Journal of Biogeography, 40, 769-777.

Hill JK, Gray MA, Khen CV, Benedick S, Tawatao N, Hamer KC (2011) Ecological impacts of tropical forest fragmentation: how consistent are patterns in species richness and nestedness? Philosophical Transactions of the Royal Society
B: Biological Sciences, 366, 3265-3276.

Hu G, Feeley KJ, Wu JG, Xu GF, Yu MJ (2011) Determinants of plant species richness and patterns of nestedness in fragmented landscapes: evidence from land-bridge islands. Landscape Ecology, 26, 1405-1417.

Li YM, Niemela J, Dianmo L (1998) Nested distribution of amphibians in the Zhoushan Archipelago, China: can selective extinction cause nested subsets of species? Oecologia, 113, 557-564.

Li ZT, Lu Z, Shu XL, Jiang GW, Xu L, Zhou F (2013) Nestedness of bird assemblages in the karst forest fragments of southwestern Guangxi, China. Chinese Birds, 4, 170-183.

Lindo Z, Winchester NN, Didham RK (2008) Nested patterns of community assembly in the colonisation of artificial canopy habitats by oribatid mites. Oikos, 117, 1856-1864.

Matthews TJ, Cottee-Jones HEW, Whittaker RJ (2015) Quantifying and interpreting nestedness in habitat islands: a synthetic analysis of multiple datasets. Diversity and Distributions, 21, 392-404.

McGarigal K, Cushman SA (2002) Comparative evaluation of experimental approaches to the study of habitat fragmentation effects. Ecological Applications, 12, 335-345.

McQuaid CF, Britton NF (2013) Host-parasite nestedness: a result of co-evolving trait-values. Ecological Complexity, $13,53-59$.

Menezes J, Fernandez F (2013) Nestedness in forest mammals is dependent on area but not on matrix type and sample size: an analysis on different fragmented landscapes. Brazilian Journal of Biology, 73, 465-470.

Morrison LW (2013) Nestedness in insular floras: spatiotemporal variation and underlying mechanisms. Journal of Plant Ecology, 6, 480-488.

Öckinger E, Smith HG (2006) Landscape composition and habitat area affects butterfly species richness in semi-natural grasslands. Oecologia, 149, 526-534.

Patterson BD, Atmar W (1986) Nested subsets and the structure of insular mammalian faunas and archipelagos. Biological Journal of the Linnean Society, 28, 65-82.

Pe'er G, Maanen C, Turbé A, Matsinos YG, Kark S (2011) Butterfly diversity at the ecotone between agricultural and semi-natural habitats across a climatic gradient. Diversity and Distributions, 17, 1186-1197.

Rodríguez-Gironés MA, Santamaría L (2006) A new algorithm to calculate the nestedness temperature of presence-absence matrices. Journal of Biogeography, 33, 924-935.

Rodríguez D, Ojeda RA (2013) Scaling coexistence and assemblage patterns of desert small mammals. Mammalian Biology, 78, 313-321.

Schouten MA, Verweij PA, Barendregt A, Kleukers RJM, de Ruiter PC (2007) Nested assemblages of Orthoptera species in the Netherlands: the importance of habitat features and life-history traits. Journal of Biogeography, 34, 1938-1946.

Simaiakis SM, Martínez-Morales MA (2010) Nestedness in centipede (Chilopoda) assemblages on continental islands (Aegean, Greece). Acta Oecologica, 36, 282-290.

Soga M, Koike S (2012) Life-history traits affect vulnerability 
of butterflies to habitat fragmentation in urban remnant forests. Ecoscience, 19, 11-20.

Soga M, Koike S (2013) Patch isolation only matters for specialist butterflies but patch area affects both specialist and generalist species. Journal of Forest Research, 18, 270-278.

Tabak MA, Poncet S, Passfield K, Martinez C (2014) Invasive species and land bird diversity on remote South Atlantic islands. Biological Invasions, 16, 341-352.

Uezu A, Beyer DD, Metzger JP (2008) Can agroforest woodlots work as stepping stones for birds in the Atlantic forest region? Biodiversity and Conservation, 17, 1907-1922.

Ulrich W, Almeida-Neto M, Gotelli NJ (2009) A consumer's guide to nestedness analysis. Oikos, 118, 3-17.

Wang SP, Zhu W, Gao X, Li XP, Yan SF, Liu X, Yang J, Gao ZX, Li YM (2014) Population size and time since island isolation determine genetic diversity loss in insular frog populations. Molecular Ecology, 23, 637-648.

Wang YP, Bao YX, Yu MJ, Xu GF, Ding P (2010) Nestedness for different reasons: the distributions of birds, lizards and small mammals on islands of an inundated lake. Diversity and Distributions, 16, 862-873.

Wang YP, Ding P, Chen SH, Zheng GM (2013) Nestedness of bird assemblages on urban woodlots: implications for conservation. Landscape and Urban Planning, 111, 59-67.
Wang YP, Wang X, Ding P (2012) Nestedness of snake assemblages on islands of an inundated lake. Current Zoology, 58, 828-836.

Worthen WB, Jones MT, Jetton RM (1998) Community structure and environmental stress: desiccation promotes nestedness in mycophagous fly communities. Oikos, 81, 45-54.

Wright DH, Patterson BD, Millelson GM, Culter A, Atmar W (1998) A comparative analysis of nested subset patterns of species composition. Oecologia, 113, 1-20.

Zhang JC, Wang YP, Jiang PP, Li P, Yu MJ, Ding P (2008) Nested analysis of passeriform bird assemblages in the Thousand Island Lake region. Biodiversity Science, 16, 321-331. (in Chinese with English abstract) [张竞成, 王彦 平, 蒋萍萍, 李鹏, 于明坚, 丁平 (2008) 千岛湖雀形目 鸟类群落嵌套结构分析. 生物多样性, 16, 321-331.]

Zhou H, Guo SH, Feng ZG (1998) Monographia of Zhejiang Islands. Higher Education Press, Beijing. (in Chinese) [周 航, 国守华, 冯志高 (1998) 浙江海岛志. 高等教育出版 社, 北京.]

Zhou Y (2000) Monograph of Chinese Butterfly. Henan Scientific and Technological Publishing House, Zhengzhou. (in Chinese) [周尧 (2000) 中国蝶类志. 河南科学和技术出 版社, 郑州.] 